\title{
Composite lymphoma of cervical lymph nodes with classical Hodgkin lymphoma and diffuse large B cell lymphoma: a case report and literature review
}

\author{
Jian Wang ${ }^{1}$, Runzhi Zhang ${ }^{2}$ \\ ${ }^{1}$ Department of Oncology, ${ }^{2}$ Department of Gynecology, The Fifth People's Hospital of Changshu, Changshu, China \\ Correspondence to: Runzhi Zhang. Department of Gynecology, The Fifth People's Hospital of Changshu, Changshu, China. \\ Email: zhangrunzhi2020@163.com.
}

\begin{abstract}
Composite lymphoma (CL) is a clinically rare entity, with unknown pathogenic mechanisms. Its diagnosis is quite difficult, and there is currently no proven treatment. In this article, we report a 64-year-old male patient presenting with swelling and mild pain in the bilateral cervical lymph nodes. Radiology revealed lesions only involved the neck. Histological examination showed that the tumor tissue had two components: mixed cellularity classical Hodgkin lymphoma (CHL-MC) and diffuse large B cell lymphoma (DLBCL). The final diagnosis was CL in the bilateral cervical lymph nodes composed of CHL-MC and DLBCL stage IA. The patient received six cycles of rituximab plus cyclophosphamide, pirubicin, vincristine, and prednisone (R-CHOP) and two cycles of rituximab maintenance therapy, and complete response (CR) was achieved. No progression was found during the 9 months of follow-up. We conducted a literature review of 28 cases of CL with CHL and DLBCL. The demography of the disease, the location of the disease involved, the subtype of CHL, the Epstein-Barr virus infection, treatment and survival were described in detail. In the discussion section, we analyzed the definition, pathogenesis, diagnosis, treatment and prognosis of CL. We hope that through case reports and literature review, we can improve the understanding of CL and optimize its treatment.
\end{abstract}

Keywords: Composite lymphoma (CL); classical Hodgkin lymphoma (CHL); diffuse large B cell lymphoma (DLBCL); case report

Submitted May 09, 2020. Accepted for publication Aug 21, 2020.

doi: 10.21037/apm-20-1290

View this article at: http://dx.doi.org/10.21037/apm-20-1290

\section{Introduction}

Composite lymphoma (CL) refers to two or more distinct types of lymphomas occurring synchronously in the same organ or anatomical site (1) and are typically composed of two B-cell lymphomas. While CLs are infrequent entities, CL with classical Hodgkin lymphoma (CHL) and diffuse large B cell lymphoma (DLBCL) is even rarer. The etiology of CL is not completely clear. It is generally believed that the occurrence of CL is closely related to the deficiency of immune function. During the management of lymphomas, the accuracy of diagnosis depends largely on the expertise and experience of pathologists, and the diagnostic results also directly affect the selection of treatment protocols.
Few reports have described CLs, and treatment decisionmaking for CL patients remains challenging. The choice of treatment for CL is mainly based on the histological type with high invasiveness. In this article, we report a 64-yearold male patient with CL with CHL-MC and DLBCL involving the bilateral cervical lymph nodes. Also, we reviewed the existing literature comprising 28 cases of CL with CHL and DLBCL, with an attempt to summarize the clinical features of this malignancy and investigate its pathogenesis, clinicopathological characteristics, diagnosis, and treatment. Compared with previous case reports, we included more comprehensive cases, and made a detailed analysis of the definition, pathogenesis, diagnosis, treatment 
and outcome of CL. We present the following article in accordance with the CARE reporting checklist (available at http://dx.doi.org/10.21037/apm-20-1290).

\section{Clinical data}

This is a case of a 64-year-old male patient of Han Chinese ethnicity. He was a retired businessman. In August 2019, the lymph nodes on both sides of his neck were incidentally found to be swollen (but without redness) and slightly painful. The patient had no fever, night sweats, or weight loss. He lived a regular life and had no family history of genetic disease. The patient had a history of hypertension, which was well controlled by oral antihypertensive drugs. Physical examination showed multiple swollen lymph nodes on both sides of the neck. The largest lymph node on the left side was $25 \mathrm{~mm} \times 14 \mathrm{~mm}$ in size, and the largest one on the right side was $18 \mathrm{~mm} \times 14 \mathrm{~mm}$ in size. They had relatively clear borders but poor mobility and were painful to the touch. On August 22, 2019, ultrasound revealed multiple swollen lymph nodes on both sides of the neck and submandibular region. On September 26, 2019, positron emission tomography-computed tomography (PET/ CT) showed the involvement of bilateral cervical, right submandibular, and anterior cervical lymph nodes, along with increased fluorodeoxyglucose (FDG) uptake (Figure 1). The possibility of lymphoma was considered. On August 27, 2019, biopsies of bilateral cervical lymph nodes were performed. Macroscopically, the lymph nodes harvested from both sides of the neck were swollen and enlarged, with firm texture and focal necrosis. Light microscopy showed the normal structures of some of the right cervical lymph nodes had been destroyed. A varying number of R-S cells were visible in tumor tissues, and they were scattered in the background of lymphocytes, eosinophils, plasma cells, tissue cells, and fibroblasts. These Reed-Sternbergcells (R-S) cells were eosinophilic and rich in the cytoplasm, with single, double, or multiple large nuclei. The nuclear membrane was thick and clear, the nuclear chromatin was finely dispersed, and the nuclei were bubble-like. There was a strongly eosinophilic large nucleolus in the center of the nucleus, surrounded by empty halos (Figure 2). Light microscopy of the left cervical lymph nodes showed that the cells were relatively large and that their nuclei were round or oval. In some cells, 2-4 nucleoli were close to the nuclear membrane; other cells had only 1 nucleolus, with multiple mitotic figures (Figure 3). Immunohistochemistry of the right-sided cervical lymphoma cells revealed the following:
CD30, +; CD15, partially +; LMP1, +; PAX5, weakly +; CD20, partially weakly +; Bcl-2, slightly +; Bcl-6, -; CD10, -; MUM1, +; CD3, -; CD5, -; (CD21, CD23) follicular dendritic network, +; BOB.1, +; Oct-2, -; C-myc, partially + ; Ki-67, +; and EBER in situ hybridization, partially + (Figure 4). This suggested the presence of a mixed cellularity classical CHL-MC on the right side of the neck. Immunohistochemistry of the left-sided cervical lymphoma cells revealed the following: CD30, +; CD15, -; LMP1, partially +; Bcl-2, partially +; CD20, +; CD79a, AX5 , +; Bcl6, -; CD10, -; MUM1, +; CD3, -; CD5, -; (CD21, CD23) follicular dendritic network, +; BOB.1, +; Oct-2, +; C-myc, +; Ki-67, + (about 60\%); and EBER in situ hybridization, partially + (Figure 5). This suggested the presence of lymph node Epstein-Barr virus (EBV)-positive DLBCL with massive necrosis on the left side of the neck. No tumor cells were seen in bone marrow aspirate. The final diagnosis was CL in the bilateral cervical lymph nodes composed of CHL-MC and DLBCL stage IA. DLBCL originated from a non-germinal center, and its tumor involved the left cervical lymph nodes, while the CHL-MC tumor involved the right cervical lymph nodes. Pathological diagnosis was particularly difficult during the diagnosis of the CL, and the malignancies needed to be distinguished from other subtypes of lymphoma. After multidisciplinary discussions, the diagnosis was finally confirmed, and a treatment plan was formulated. Six cycles of rituximab plus cyclophosphamide, pirubicin, vincristine, and prednisone (R-CHOP) regimen consisting of rituximab $622.5 \mathrm{mg}$ on day 1 , cyclophosphamide $1,245 \mathrm{mg}$ on day 2 , epirubicin $116 \mathrm{mg}$ on day 2 , vincristine $2 \mathrm{mg}$ on day 2 , and prednisolone $100 \mathrm{mg}$ on days 2-6 (repeated every 3 weeks), were started on October 9, 2019. On December 16, 2019 (after the third cycle), CT showed that there were no swollen lymph nodes in the neck, and the treatment response was therefore evaluated as "unconfirmed complete response" (CRu). On March 20, 2020 (after the six-cycle treatment was completed), PET/CT showed that the multiple swollen cervical lymph nodes had significantly shrank or reduced in size, and there was no increase in FDG uptake (Figure 1). The growth of tumors had obviously been suppressed, and CR was achieved. After two sessions of multidisciplinary discussions, two further cycles of rituximab alone were used as maintenance treatment. The last treatment was performed on April 22, 2020. The patient's tolerance and compliance were good during the treatment, and no grade III/IV toxicities occurred. The treatment has since been discontinued, and no obvious signs 

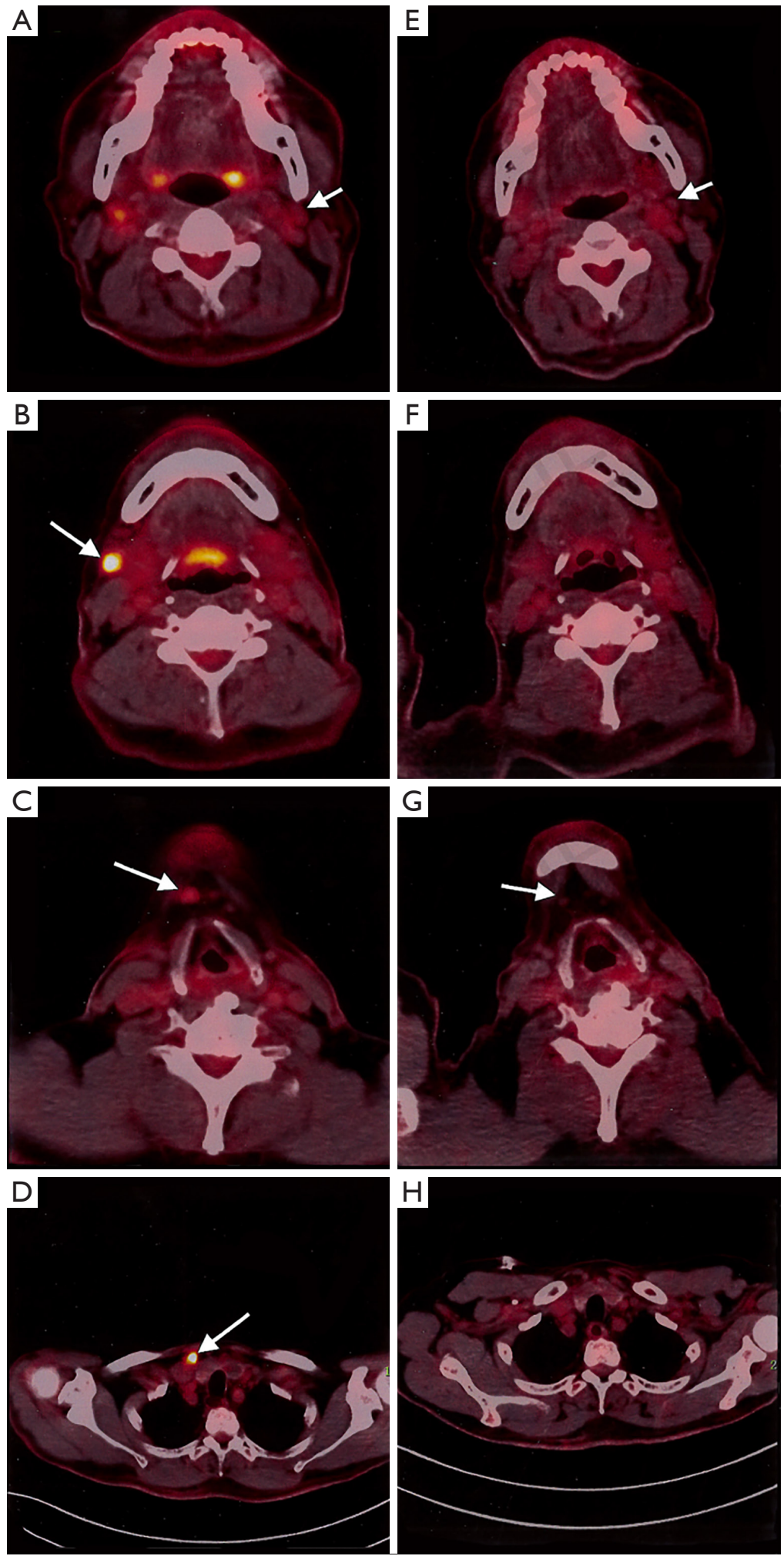

Figure 1 PET/CT before treatment on September 26, 2019 showed the involvement of bilateral cervical (A,B), right submandibular (C), and anterior cervical (D) lymph nodes, along with increased FDG uptake and PET/CT after treatment on March 20, 2020 showed that the multiple swollen cervical lymph nodes had significantly shrank or reduced in size, and there was no increase in FDG uptake (E,F,G,H). Arrows: location of tumor. 

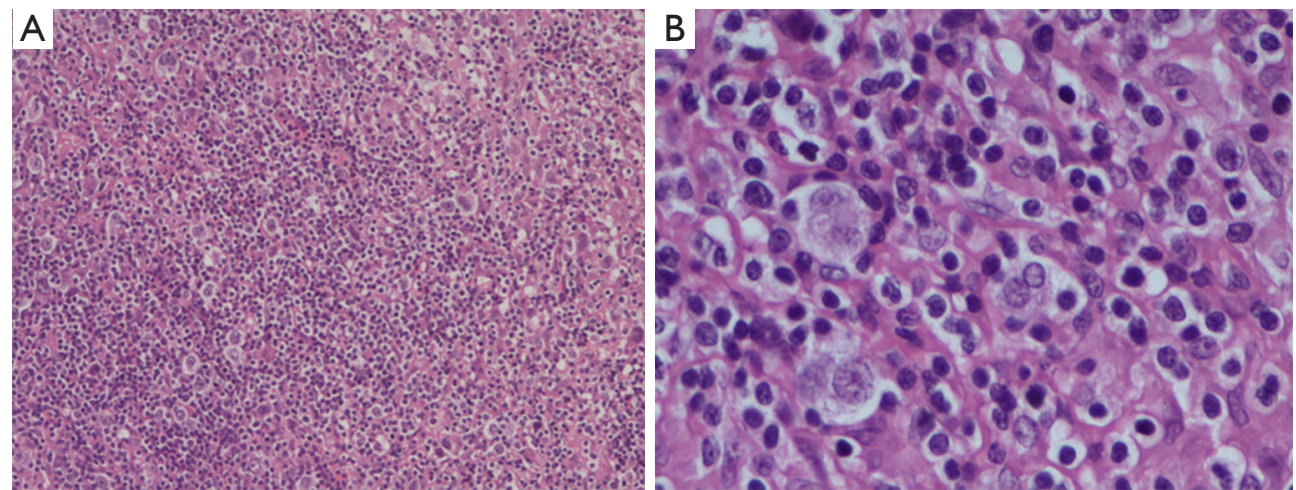

Figure 2 Hematoxylin and eosin of classical Hodgkin's lymphoma of the cervical lymph node. (A) Mixed lymphocyte, eosinophil granulocyte and neutrophil granulocyte infiltrating the cervical lymph node (100x); (B) Hodgkin and Reed-Sternberg (RS) cells are present (400x).
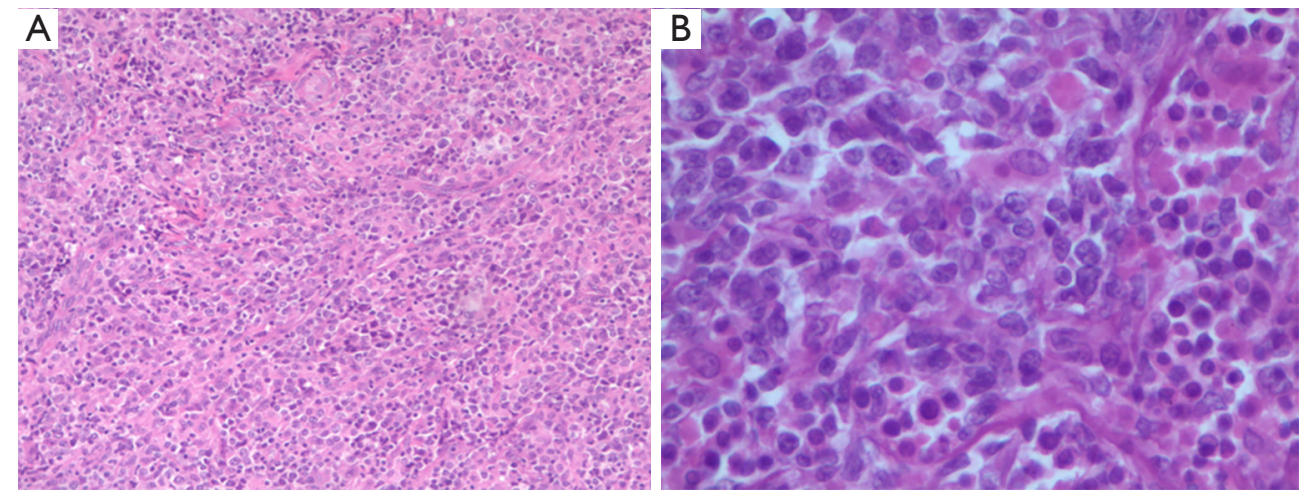

Figure 3 Hematoxylin and eosin of diffuse large B-cell lymphoma of the cervical lymph node. (A) Large lymphoid cells diffusely infiltrating the cervical lymph node (100x); (B) nucleoli and frequent mitotic figures (400x).

of recurrence were seen 9 months after the medication was initiated. The milestone events during diagnosis and treatment are summarized in Table 1.

All procedures performed in studies involving human participants were in accordance with the ethical standards of the institutional and/or national research committee(s) and with the Helsinki Declaration (as revised in 2013). Written informed consent was obtained from the patient for publication of this case report and any accompanying images.

\section{Literature review}

Literature review was performed by searching PubMed using keywords including "composite", "lymphoma", "Hodgkin", and "diffuse large B-cell" to analyze the CL cases with CHL and DLBCL components. Based on the retrieved articles, the sex, age, diagnosis, biopsy site, CHL subtype, EBV status, clonal relationship between individual components, treatment, and prognosis were systematically analyzed.

A total of 28 CL cases with CHL and DLBCL (including our current case) entered the final analysis (Table 2). Among these patients, there were 13 males and 12 females, with the sex being unknown in 3 cases. Except for 3 patients with unknown age, the average age of the remaining 25 cases at the time of presentation was 51.8 years ( $6-79$ years; median: 58 years). The lymph node was the most frequently affected site $(\mathrm{n}=18,64 \%)$, followed by the mediastinum $(\mathrm{n}=3)$, spleen $(n=3)$, intestine or mesentery $(n=3)$, stomach $(n=1)$, heart valve $(n=1)$, lung parenchyma $(n=1)$, tonsils $(n=1)$, and subcutaneous $(\mathrm{n}=1)$. The CHL subtypes included nodular sclerosis CHL (NS-CHL) ( $\mathrm{n}=15,54 \%)$, mixed cell subtypes ( $\mathrm{n}=9,32 \%)$, and lymphocyte-depleted CHL (LD-CHL) 

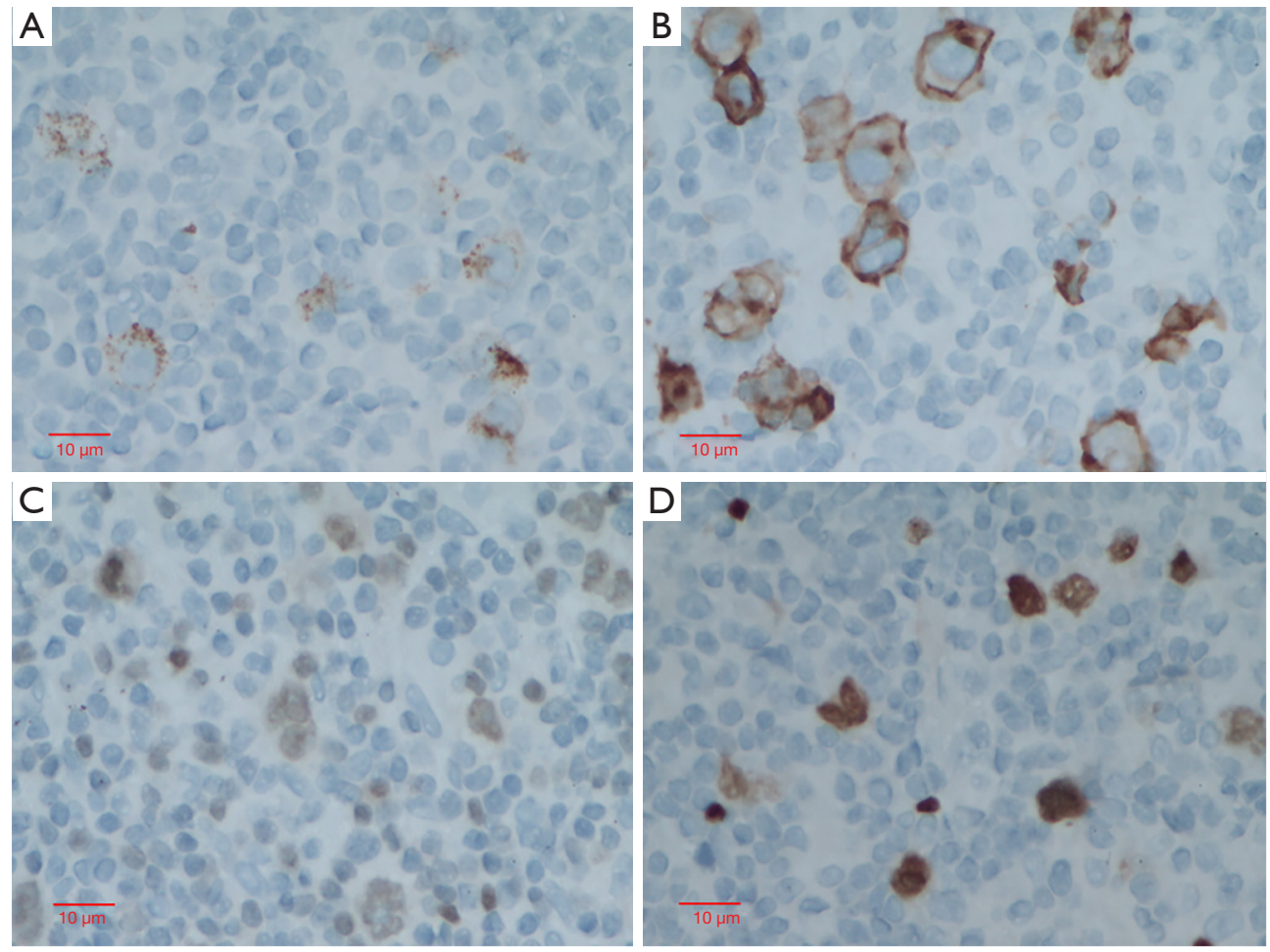

Figure 4 Immunoperoxidase stain of classical Hodgkin's lymphoma of the cervical lymph node. (A) Neoplastic cells diffusely partial positive for CD15 (400x); (B) neoplastic cells diffusely positive for CD30 (400×); (C) neoplastic cells diffusely positive for MUM1 (400x); (D) neoplastic cells diffusely weakly positive for PAX5 (400x).
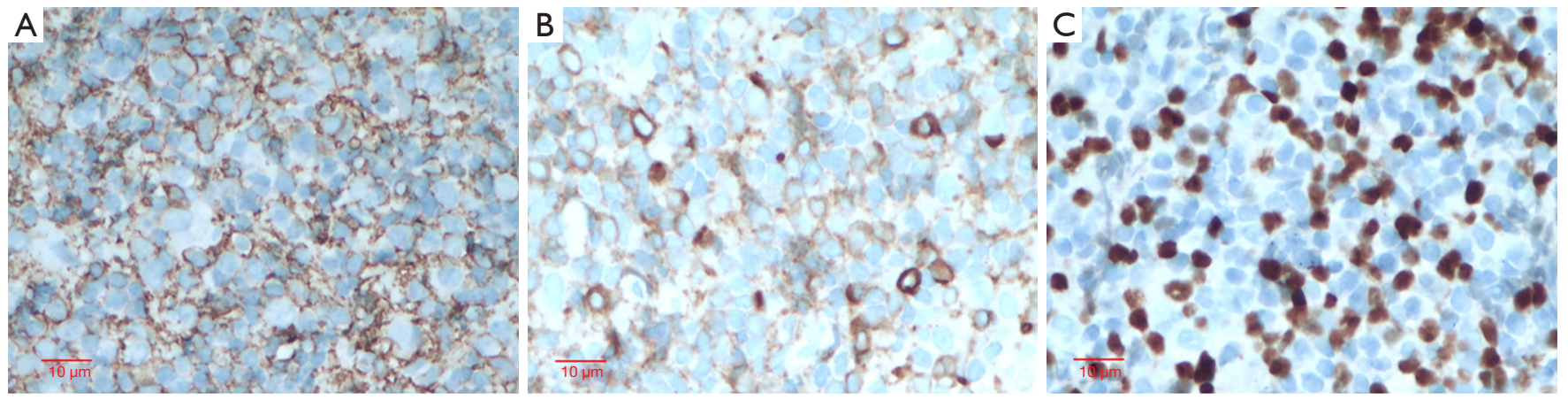

Figure 5 Immunoperoxidase stain of diffuse large B-cell lymphoma of the cervical lymph node. (A) Neoplastic cells diffusely positive for CD20 (400×); (B) neoplastic cells diffusely positive for CD79a (400×); (C) neoplastic cells diffusely positive for PAX5 (400×).

$(\mathrm{n}=1,4 \%)$. Furthermore, 7 (25\%) of the 22 specimens tested positive for EBV, among which EBV was positive in both CL components in 5 cases, in CHL alone in 1 case, and in DLBCL alone in 1 case. IGH gene rearrangement in six cases (21\%) showed a complete or partial clonal relationship between these two components. Among the reported cases, $21(75 \%)$ received chemotherapy (CHOP/R-CHOP or similar regimens in most cases), 6 (21\%) underwent surgery, $1(4 \%)$ received radiotherapy, and $2(7 \%)$ were treated with autologous stem cell transplantation. Follow-up data were available in 22 of the 28 patients. The average followup period was 19 months (range: 1-48 months; median: 14 months), during which 10 died. The average survival time was 3 months (range: 1-6 months; median: 
Table 1 The milestone events during diagnosis and treatment of our current case

\begin{tabular}{|c|c|}
\hline Time & Event \\
\hline August 22, 2019 & Ultrasound revealed multiple swollen lymph nodes on both sides of the neck and submandibular region \\
\hline August 27, 2019 & Biopsies of bilateral cervical lymph nodes were performed \\
\hline September 26, 2019 & PET/CT showed the involvement of bilateral cervical, right submandibular, and anterior cervical lymph nodes \\
\hline October 30, 2019 & The second cycle of R-CHOP treatment \\
\hline November 26, 2019 & The third cycle of R-CHOP treatment \\
\hline December 16, 2019 & The treatment response as evaluated by CT was CRu \\
\hline December 19, 2019 & The fourth cycle of R-CHOP treatment \\
\hline March 20, 2020 & The treatment response as evaluated by PET/CT was CR \\
\hline March 30, 2020 & The first cycle of rituximab maintenance treatment \\
\hline April 22, 2020 & The second cycle of rituximab maintenance treatment \\
\hline
\end{tabular}

$\mathrm{PET} / \mathrm{CT}$, positron emission tomography-computed tomography; CRu, unconfirmed complete response; CR, complete response.

3 months) in 4 EBV-positive cases and 15 months (range: 626 months; median: 21 months) in $4 \mathrm{EBV}$-negative cases.

\section{Discussion}

In 1952, Custer was first to propose the concept of CL, which referred to the development of more than one histological variety of lymphoma in a single patient (2). This definition was later extended by Hicks et al. (3) and perfected by Kim et al. (1), who described CL as a tumor with the synchronous presence of two or more different types of lymphoma in the same organ or anatomical site. Most cases of CL involve two distinct types of B-cell lymphomas, usually a low-grade B-cell lymphoma (e.g., follicular lymphoma and mantle cell lymphoma; or, chronic lymphocytic leukemia and follicular lymphoma). They have a common germinal-center B-cell precursor. The combination of DLBCL and CHL is a rare type of CL (2). The co-existence of CHL and nodular lymphocytepredominant Hodgkin lymphoma (NLPHL) has also been reported (4).

Due to the diversity and complexity of lymphomas and the varied causes of different lymphoma types, no single definite mechanism has been available to explain the pathogenesis of different types of CL. It is generally believed that the occurrence of CL is closely related to immunodeficiency. It is more likely to occur in situations of lymphoproliferative disease (e.g., Castleman's disease), immunosuppressive status, high-dose chemotherapy, and multiple viral infections $(5,6)$.

Lymphoma was thought to involve the monoclonal proliferation of lymphocytes (including T cells, B cells or NK cells), whereas polyclonal proliferation was often considered to be a reactive lymphoid proliferation. However, the emergence of CL questioned these traditional understandings. Studies have shown that polyclonality of a lesion is not necessarily a reactive proliferation, and two different morphological types or phenotypes do not always represent two different lymphomas with unrelated clones. It has been argued that CLs represent different phases of clonal evolution and not a coincidental simultaneous occurrence of two unrelated lymphomas. As time elapses, lymphomas often evolve from the small-cell type to the large-cell type or from the follicular pattern to the diffuse pattern (7). The transition from NLPHL to DLBCL has also been reported (8). Some authors also believe that the change of lymphomas with time is actually the progression of the disease rather than a CL. In such cases, all malignant cells will eventually become more aggressive over time as part of their natural course. It has also been proposed that 


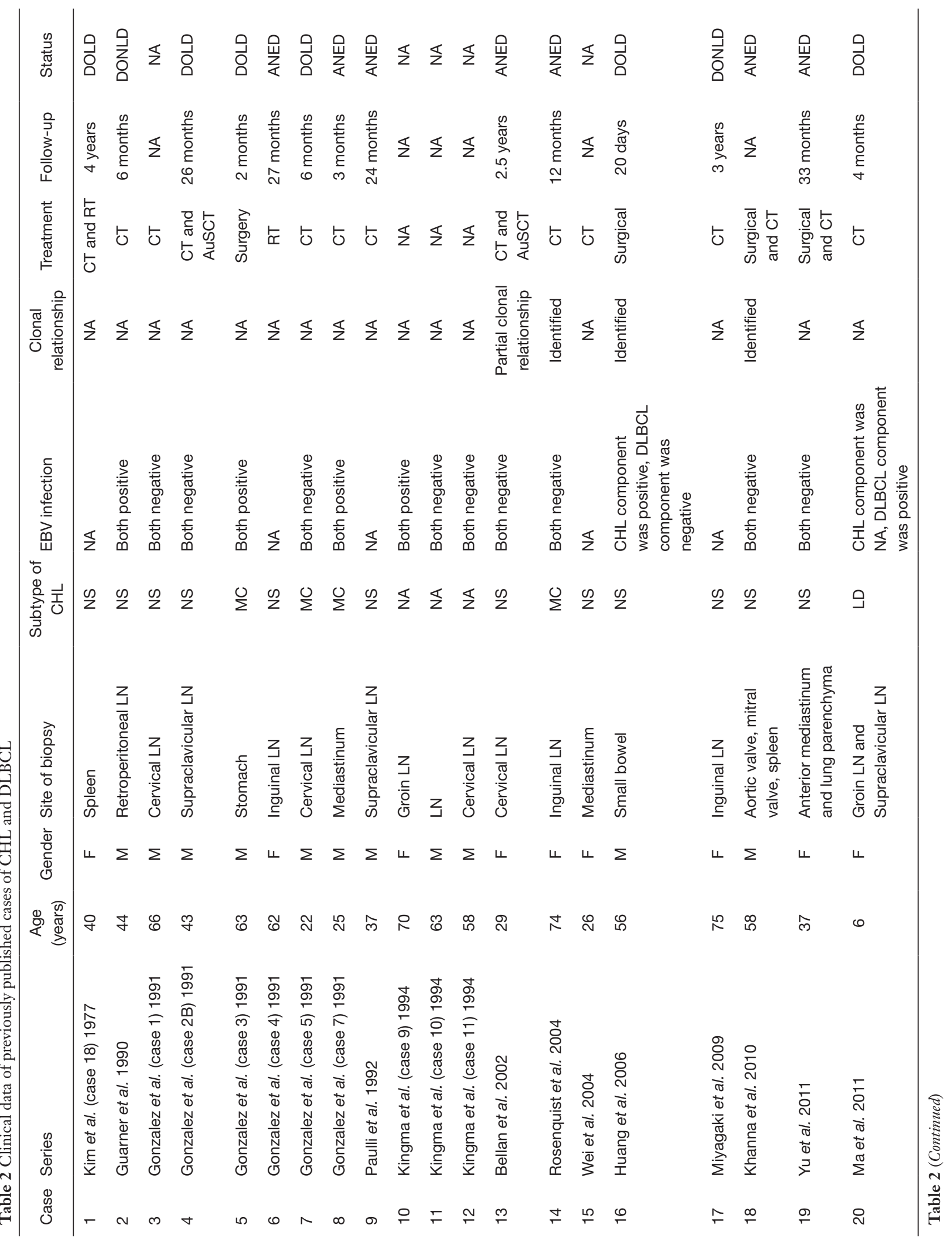




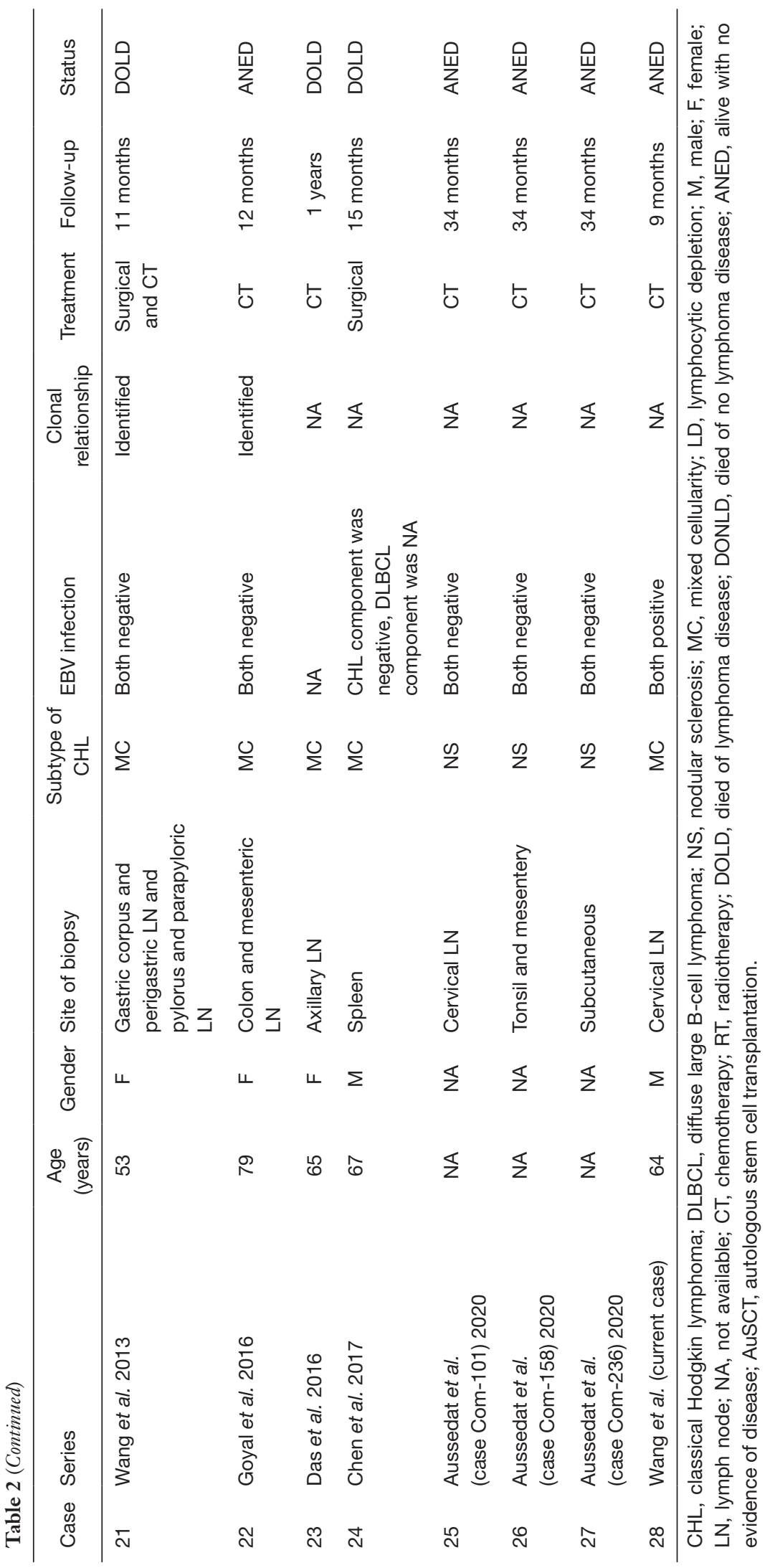


the histological components of a CL must have distinctly different immunophenotypes and molecular lesions. These controversies attest to a lack of sufficient understanding of the pathogenesis of these lymphomas or the heterogeneity between Hodgkin lymphoma (HL) and non-Hodgkin lymphoma (NHL). The complex interrelationship of the lymphoid system and its clonal evolution require further investigation.

CL is rarely described in literature, and its description has been limited to isolated case reports (9-12). However, its incidence may be underestimated as sophisticated techniques are required in the diagnosis of CL, and, due to its inconsistent definitions, the incidence of CL reported across different studies varies (13). A study carried out in over 1,000 NHL cases found the incidence of CL ranged from $1 \%$ to $4.7 \%$ (7), but depended on the experience of clinicians and the typing systems they applied. Based on data from patients undergoing exploratory laparotomy, Kim et al. concluded that the incidence of CL was 3.5\% (3/84) (14). The CL patients enrolled in Maeshima et al.'s study included both synchronous and successive lymphoma cases, whereas the number of actual CL cases was small (13).

CL is extremely rare, and its diagnosis is mainly based on morphology. According to Kim et al., the most important step in identifying CL under the microscope is to carefully examine the diseased tissue under a low-power microscope and search for different lesions between two involved nodules or in abnormal nodules (14). When a lesion affects the entire spleen or lymph nodes, some seemingly insignificant and morphologically different sites are often neglected. Because the more aggressive component usually dominates, it can mask other components. These lesions are recognized only when the original materials are reviewed after a second biopsy reveals the presence of histological changes. However, due to the complexity of CL components and the wide morphological overlap between (or among) the cells of two or more components, morphological diagnosis alone often leads to misdiagnosis. Immunohistochemistry can improve diagnostic specificity and identify both clonality and cell subtypes (15). New molecular techniques using polymerase chain reaction (PCR) to detect the immunoglobulin heavy chain $(\mathrm{IgH})$ and $\mathrm{T}$ cell receptor gene rearrangement can confirm the clonality of B-cell and T-cell components and further help diagnose CL (16). Flow cytometry can simultaneously distinguish different cell phenotypes and determine the clonality of different cell populations. Demurtas et al. successfully identified 17 CL cases in 1,332 patients with lymphomas by flow cytometry (17). Other reliable detection methods include cytogenetics, fluorescence in situ hybridization (FISH), DNA sequencing, and cDNA microarray. Laser capture microdissection (LCM) has particularly high accuracy (2), and when LCM is used to evaluate clonal gene rearrangement, chromosomal translocation, and gene sequencing on the cells of two tumor components, it not only increases the diagnostic accuracy but also enables genetic research on the origin of these two different tumor cells.

The term "CL" was initially used to describe the presence of more than one type of lymphoma in a patient. Today, this term is limited to the infrequently identified phenomenon of two or more lymphoma clones with distinct morphologies and immunophenotypes at one anatomic site (i.e., one organ or one tissue) (18). However, some new immunohistological and molecular biological techniques have revealed that many so-called CLs do not necessarily meet this definition. In fact, these "CLs" only represent two different morphological phenotypes of a single malignant clone, and the presence of two different morphologies or phenotypes does not always represent two types of clonally unrelated lymphomas $(19,20)$. In many of these cases, the so-called CLs may be transformed from one clone or derived from one common precursor cell type. In most cases, the proliferation of two different types of cells in a CL is clonally related, suggesting they have a common lymphoid progenitor cell. It is also possible that the two proliferations that lead to composite injuries have different cellular sources, as supported by $\mathrm{IgH}$ rearrangements and by somatic mutations found in two clones (21). Some composite lesions are considered to be the invasive transformations of indolent lymphomas; however, such clonal evolution can be regarded as disease progression with time rather than CL. Many researchers now believe that the definition of CL has changed, and the differences in the immunophenotypic patterns and clones should be identified between two lymphoproliferative lesions (22). Histological and immunophenotyping studies support two or more distinct lymphoid hyperplasias but cannot identify their clonal relationship. Only the genetic rearrangement of heavy chains and/or the cytogenetic abnormalities can be used to distinguish the common or different origins of the relevant pathological processes (23).

Histopathologically, HL is clearly different from NHL; however, most studies have confirmed that most CLs with CHL and NHL, including follicular lymphoma, mantle cell lymphoma, DLBCL, and chronic lymphocytic leukemia, 
are clonally related (24). Somatic mutation of IGH V gene in R-S cells, a marker of the germinal-center B cells and their descendants, has been detected in most isolated CHL cases (25-27), suggesting that these cells or their precursors are derived from B cells (28). Unfortunately, we were not able to perform further molecular biological studies on our current case to determine whether there is a clonal relationship between CHL cells and DLBCL cells.

It has been proposed that EBV infection has played an important role in the pathogenesis of CL with CHL and DLBCL. EBV infection causes primary immunodeficiency and viremia, and further induces the malignant transformation and proliferation of lymphocytes through anti-apoptotic factors and cytokinins (29). EBVpositive DLBCL in elderly patients may arise due to agerelated immune impairment caused by the progressive deterioration of immunity (30). About $40 \%$ of solitary CHL cases have been found to occur with EBV infection, which might be involved in the tumorigenesis $(31,32)$. In contrast, the incidence of EBV-positive DLBCL is much lower: about $8.7-11.4 \%$ in eastern Asian and $<5 \%$ in Western countries (33). Goyal et al. reviewed 21 CL cases with CHL and DLBCL and found EBV was positive in both components in $31 \%$ of CL cases, suggesting a shared origin of these two components from a common B-cell precursor (34). Due to technical limitations, we were unable to detect the clonal relationship between the two tumor components in our current case; however, immunohistochemistry suggested that $\mathrm{EBV}$ was positive in both the CHL and DLBCL cells, which suggests that the two components in this patient might originate from the same clone. Notably, clonal relatedness of two tumors cannot be ruled out if only one type of lymphoma is EBV-positive, because EBV infection or reactivation may occur at the same time or after CHL transformation. As shown by in situ hybridization and immunohistochemical tests, EBV was negative in some CL with DLBCL and CHL specimens, which indicates EBV infection may not be essential for the onset of this CL type, although the relevant mechanisms still need to be examined further.

Studies have shown that EBV infection is significantly correlated with poor prognosis in CL patients $(34,35)$. According to our literature review, 10 of the $28 \mathrm{CL}$ with CHL and DLBCL patients died, 4 of whom were EBVpositive, 4 of whom were EBV-negative, and 2 of whom had unknown EBV status. The average survival time of EBV-positive patients was significantly shorter than that of EBV-negative patients (3 vs. 15 months), suggesting
EBV infection may be an adverse prognostic factor for CL. However, this also requires more extensive research.

The treatment strategy of CL should consider both tumor components. Existing data indicate that the biological behaviors of two or more components of CL are similar to those of the solitary diseases. If different lymphomas occur sequentially, each disease should be treated according to its own therapeutic principles $(19,36)$. The management of CL with two components occurring synchronously is more challenging: the prognosis of patients with CL depends on the more aggressive component, and the choice of treatment protocol is mainly based on the more invasive histological type (37). For CL with CHL and DLBCL, DLBCL is more invasive. Thus, the R-CHOP regimen, supplemented with surgery, radiotherapy, and autologous stem cell transplantation, is a reasonable strategy. If immunohistochemistry of tumor cells suggests CD20 +, targeted therapy with rituximab will achieve satisfactory efficacy.

\section{Conclusions}

We reported a case of CL with CHL and DLBCL and reviewed the relevant literature. The diagnosis of CL is difficult and must be cautiously confirmed by immunophenotyping and/or molecular biology tests. Different combinations of CLs may have different natural histories, prognoses, and therapies. A better understanding of CL will help to optimize the treatments. In addition, research on such cases can provide clues to the etiology and interrelationship of the evolution of lymphoma clones.

\section{Acknowledgments}

We would like to thank the patient and his family for their consent in publishing this case report.

Funding: The present study was supported by the Science and Technology Development Project of Healthy and Family Planning Commission of Changshu (No. csws201705).

\section{Footnote}

Reporting Checklist: The authors have completed the CARE reporting checklist. Available at http://dx.doi.org/10.21037/ apm-20-1290

Conflicts of Interest: Both authors have completed the 
ICMJE uniform disclosure form (available at http:// dx.doi.org/10.21037/apm-20-1290). The authors report grants from Healthy and Family Planning Commission of Changshu, during the conduct of the study.

Ethical Statement: The authors are accountable for all aspects of the work in ensuring that questions related to the accuracy or integrity of any part of the work are appropriately investigated and resolved. Written informed consent was obtained from all patients. All procedures performed in studies involving human participants were in accordance with the ethical standards of the institutional and/or national research committee(s) and with the Helsinki Declaration (as revised in 2013). Written informed consent was obtained from the patient for publication of this case report and any accompanying images.

Open Access Statement: This is an Open Access article distributed in accordance with the Creative Commons Attribution-NonCommercial-NoDerivs 4.0 International License (CC BY-NC-ND 4.0), which permits the noncommercial replication and distribution of the article with the strict proviso that no changes or edits are made and the original work is properly cited (including links to both the formal publication through the relevant DOI and the license). See: https://creativecommons.org/licenses/by-nc-nd/4.0/.

\section{References}

1. Kim H, Hendrickson R, Dorfman RF. Composite lymphoma. Cancer 1977;40:959-76.

2. Mokhtar NM. Review article composite lymphoma. J Egypt Natl Canc Inst 2007;19:171-5.

3. Hicks EB, Rappaport H, Winter WJ. Follicular lymphoma; a re-evaluation of its position in the scheme of malignant lymphoma, based on a survey of 253 cases. Cancer 1956;9:792-821.

4. Küppers R, Dührsen U, Hansmann ML. Pathogenesis, diagnosis, and treatment of composite lymphomas. Lancet Oncol 2014;15:e435-46.

5. Carbone A, Gloghini A. AIDS-related lymphomas: from pathogenesis to pathology. Br J Haematol 2005;130:662-70.

6. Tsitsipatis D, Gorospe M. 'RNA circles of influence' in Kaposi sarcoma. Ann Transl Med 2019;7:S109.

7. Kim H. Composite lymphoma and related disorders. Am J Clin Pathol 1993;99:445-51.

8. Cotta CV, Coleman JF, Li S, et al. Nodular lymphocyte predominant Hodgkin lymphoma and diffuse large B-cell lymphoma: a study of six cases concurrently involving the same site. Histopathology 2011;59:1194-203.

9. Sakakibara A, Kohno K, Iwakoshi A, et al. Diagnostic utility of programmed cell death ligand 1 (clone SP142) in mediastinal composite lymphoma: A report of two cases. Pathol Int 2020;70:116-22.

10. Sabater-Marco V, Santonja-López N, Ortíz-Zuluaga S, et al. Orbital soft tissue composite lymphoma presenting as recurrence of a nodal lymphoma with mantle and follicular cell components: A case report, literature review and guideline for the treatment of patients. Rev Esp Patol 2020;53:48-54.

11. Sharma S, Singh V, Bisaria D, et al. Composite lymphoma comprising mantle cell lymphoma and Epstein-Barr virus positive classic Hodgkin lymphoma: A rare case. Indian J Pathol Microbiol 2019;62:488-90.

12. González-Gascón YMI, Menarguez J, Kwon M, et al. Composite Lymphoma Containing Mantle Cell and Peripheral T-cell Lymphoma, Not Otherwise Specified: A Report of 2 Cases Treated With Up-front Autologous Stem Cell Transplantation. Appl Immunohistochem Mol Morphol 2019. [Epub ahead of print].

13. Maeshima AM, Taniguchi H, Nomoto J, et al. Clinicopathological features of classical Hodgkin lymphoma in patients $\geq 40$ years old, with special reference to composite cases. Jpn J Clin Oncol 2015;45:921-8.

14. Kim H, Dorfman RF. Morphological studies of 84 untreated patients subjected to laparotomy for the staging of non-Hodgkin's lymphomas. Cancer 1974;33:657-74.

15. Raufi A, Jerkins J, Lyou Y, et al. A Patient with Supraclavicular Lymphadenopathy and Anterior Mediastinal Mass Presenting as a Rare Case of Composite Lymphoma: A Case Report and Literature Review. Case Rep Oncol 2016;9:854-60.

16. Suefuji N, Niino D, Arakawa F, et al. Clinicopathological analysis of a composite lymphoma containing both $\mathrm{T}$ - and B-cell lymphomas. Pathol Int 2012;62:690-8.

17. Demurtas A, Aliberti S, Bonello L, et al. Usefulness of multiparametric flow cytometry in detecting composite lymphoma: study of 17 cases in a 12-year period. Am J Clin Pathol 2011;135:541-55.

18. Sanchez S, Holmes H, Katabi N, et al. Composite lymphocyte-rich Hodgkin lymphoma and peripheral T-cell lymphoma associated with Epstein-Barr virus: a case report and review of the literature. Arch Pathol Lab Med 2006;130:107-12.

19. Marafioti T, Hummel M, Anagnostopoulos I, et al. 
Classical Hodgkin's disease and follicular lymphoma originating from the same germinal center B cell. J Clin Oncol 1999;17:3804-9.

20. Assaf C, Hummel M, Dippel E, et al. Common clonal T-cell origin in a patient with T-prolymphocytic leukaemia and associated cutaneous T-cell lymphomas. $\mathrm{Br}$ J Haematol 2003;120:488-91.

21. Badea M, Dobrea C, Badea D, et al. The composite lymphoma: chronic lymphocytic leukemia--classic Hodgkin's lymphoma. Rom J Morphol Embryol 2010;51:353-8.

22. Tsimberidou AM, O'Brien S, Khouri I, et al. Clinical outcomes and prognostic factors in patients with Richter's syndrome treated with chemotherapy or chemoimmunotherapy with or without stem-cell transplantation. J Clin Oncol 2006;24:2343-51.

23. Jaffe ES, Zarate-Osorno A, Kingma DW, et al. The interrelationship between Hodgkin's disease and nonHodgkin's lymphomas. Ann Oncol 1994;5 Suppl 1:7-11.

24. Schneider S, Crescenzi B, Schneider M, et al. Subclonal evolution of a classical Hodgkin lymphoma from a germinal center B-cell-derived mantle cell lymphoma. Int J Cancer 2014;134:832-43.

25. Marafioti T, Hummel M, Foss HD, et al. Hodgkin and reed-sternberg cells represent an expansion of a single clone originating from a germinal center B-cell with functional immunoglobulin gene rearrangements but defective immunoglobulin transcription. Blood 2000;95:1443-50.

26. Kanzler H, Küppers R, Hansmann ML, et al. Hodgkin and Reed-Sternberg cells in Hodgkin's disease represent the outgrowth of a dominant tumor clone derived from (crippled) germinal center B cells. J Exp Med 1996;184:1495-505.

27. Hummel M, Marafioti T, Stein H. Clonality of ReedSternberg cells in Hodgkin's disease. N Engl J Med 1999;340:394-5.

28. De Silva NS, Klein U. Dynamics of B cells in germinal

Cite this article as: Wang J, Zhang R. Composite lymphoma of cervical lymph nodes with classical Hodgkin lymphoma and diffuse large B cell lymphoma: a case report and literature review. Ann Palliat Med 2020;9(5):3651-3662. doi: 10.21037/apm-201290 centres. Nat Rev Immunol 2015;15:137-48.

29. Bellan C, Lazzi S, Zazzi M, et al. Immunoglobulin gene rearrangement analysis in composite hodgkin disease and large B-cell lymphoma: evidence for receptor revision of immunoglobulin heavy chain variable region genes in Hodgkin-Reed-Sternberg cells? Diagn Mol Pathol 2002;11:2-8.

30. Hwang YY, Leung AY, Lau WH, et al. Synchronous Epstein-Barr virus-positive diffuse large B-cell lymphoma of the elderly and Epstein-Barr virus-positive classical Hodgkin lymphoma. Histopathology 2011;59:352-5.

31. Schmitz R, Renné C, Rosenquist R, et al. Insights into the multistep transformation process of lymphomas: IgHassociated translocations and tumor suppressor gene mutations in clonally related composite Hodgkin's and non-Hodgkin's lymphomas. Leukemia 2005;19:1452-8.

32. Küppers R. Molecular biology of Hodgkin lymphoma. Hematology Am Soc Hematol Educ Program 2009:491-6.

33. Ok CY, Papathomas TG, Medeiros LJ, et al. EBV-positive diffuse large B-cell lymphoma of the elderly. Blood 2013;122:328-40.

34. Goyal G, Nguyen AH, Kendric K, et al. Composite lymphoma with diffuse large B-cell lymphoma and classical Hodgkin lymphoma components: A case report and review of the literature. Pathol Res Pract 2016;212:1179-90.

35. Lu TX, Liang JH, Miao Y, et al. Epstein-Barr virus positive diffuse large B-cell lymphoma predict poor outcome, regardless of the age. Sci Rep 2015;5:12168.

36. de Leval L, Vivario M, De Prijck B, et al. Distinct clonal origin in two cases of Hodgkin's lymphoma variant of Richter's syndrome associated With EBV infection. Am J Surg Pathol 2004;28:679-86.

37. Szczepanowski M, Masqué-Soler N, Oschlies I, et al. Composite lymphoma of nodular lymphocyte-predominant and classical Hodgkin lymphoma-Epstein-Barr virus association suggests divergent pathogenesis despite clonal relatedness. Hum Pathol 2013;44:1434-9. 